\title{
A ToxR-dependent role for the putative phosphoporin VCA1008 in bile salt resistance in Vibrio cholerae El Tor N16961
}

Correspondence

Wanda Maria Almeida von Krüger wmavk@biof.ufrj.br

Received 1 July 2010

Accepted 2 August 2010

\author{
Carolina Lage Goulart, Guilherme Garcia dos Santos, \\ Livia Carvalho Barbosa, Letícia Miranda Santos Lery, \\ Paulo Mascarello Bisch and Wanda Maria Almeida von Krüger
}

Unidade Multidisciplinar de Genômica, Instituto de Biofísica Carlos Chagas Filho, Universidade Federal do Rio de Janeiro, Rio de Janeiro, Brazil

\begin{abstract}
The putative phosphoporin encoded by vca1008 of Vibrio cholerae 01 is expressed in vivo during infection and is essential for the intestinal colonization of infant mice. In vitro, its expression is induced under inorganic phosphate $\left(P_{i}\right)$ limitation in a PhoB/R-dependent manner. In this work we demonstrated that VCA1008 has a strain-specific role in the physiology and pathogenicity of V. cholerae O1. Disruption of vca1008 led to a growth defect, an inability to colonize and a high susceptibility to sodium deoxycholate (DOC; the major bile compound) in the El Tor biotype strain N16961, but did not affect the classical strain 0395 in the same way. Furthermore, vca1008 promoter activity was higher in $\mathrm{N} 16961$ cells grown under a low $\mathrm{P}_{\mathrm{i}}$ supply in the presence of DOC than in the absence of the detergent. In the $\mathrm{P}_{\mathrm{i}}$-limited cells, vca1008 was positively regulated by PhoB, but when DOC was added to the medium, it negatively affected the PhoB-mediated activation of the gene, and enhanced vca1008 expression in a ToxR-dependent manner. These findings reveal for the first time a complex strain-specific interplay between ToxR and PhoB/R systems to control porin genes, as well as the influence of DOC on the expression of PhoB-and ToxR-regulated genes and pathogenesis in pandemic strains of $V$. cholerae.
\end{abstract}

\section{INTRODUCTION}

Vibrio cholerae is a Gram-negative bacterium found freeliving or in association with phytoplankton, zooplankton, crustaceans and molluscs in estuarine and other aquatic environments (Wai et al., 1999). Several strains of the serogroups $\mathrm{O} 1$ and $\mathrm{O} 139$ are human pathogens and cause cholera, an often-fatal diarrhoeal disease. In the various niches of the human gastrointestinal tract, the bacterium faces distinct conditions with respect to acidity, osmolarity, oxygen levels and nutrient sources (Chowdhury et al., 1996). V. cholerae reaches the small intestine, where it expresses virulence factors, despite the presence of inhibitory substances such as bile (Reidl \& Klose, 2002), a digestive secretion constituted of bile salts, phospholipids, cholesterol and the pigment biliverdin (Begley et al., 2005; Gunn, 2000). Bile acts as a biological detergent that emulsifies and solubilizes lipids and, as such, possesses antimicrobial activity that is attributed mainly to its ability to disrupt cell-membrane integrity (reviewed by Begley et al., 2005). Enteric bacteria respond to bile by altering the expression of genes whose products are involved in signal sensing and transduction, in general stress adaptation

Abbreviations: DOC, sodium deoxycholate; OMP, outer membrane protein.
(Bernstein et al., 1999; Leverrier et al., 2003; Savijoki et al., 2005; van Velkinburgh \& Gunn, 1999) and in bile resistance mechanisms (Gänzle et al., 1999; Gupta \& Chowdhury, 1997; Krukonis \& Dirita, 2003; Pace et al., 1997; Provenzano \& Klose, 2000; van Velkinburgh \& Gunn, 1999).

Bile salts can enter the cytoplasm of Gram-negative bacterial cells through the hydrophilic channels of outer membrane porins (Gunn, 2000; Thanassi et al., 1997) or across the membrane bilayer in uncharged forms (Plésiat \& Nikaido, 1992). TolC-dependent and -independent active efflux mechanisms pump bile acids out of cells (Bina \& Mekalanos, 2001; Chatterjee et al., 2004; Gunn, 2000; Poole, 2002; Thanassi et al., 1997), but lipopolysacharide (LPS) and porins also play roles vis-à-vis intrinsic bile salt resistance in Gram-negative bacteria (Gunn, 2000; Snyder \& McIntosh, 2000). The involvement of pore-forming channels in resistance to bile salts is mainly due to the wide flexibility of porin expression (Achouak et al., 2001). In many cases, acquired resistance in Gram-negative bacteria can be attributed to loss of porins or alterations in their expression (Poole, 2002).

In $V$. cholerae, the acquired resistance to bile in vitro can result from a synergy between intrinsic resistance mechanisms and ToxR modulation of the expression of OmpU 
and OmpT porins (Provenzano et al., 2000). Bile stimulates transcription of ompU and cells expressing OmpU alone are more resistant to bile in vitro than those expressing OmpT alone (Provenzano et al., 2001; Provenzano \& Klose, 2000). Apparently, the protection imparted to V. cholerae cells by OmpU is due to its higher cation selectivity and impermeability to sodium deoxycholate (DOC; the major component of bile salts) in comparison with OmpT (Duret \& Delcour, 2006; Simonet et al., 2003). However, neither OmpU nor OmpT is essential for expression of virulence factors in vitro or intestinal colonization (Osorio et al., 2004).

The product of $v c a 1008$ has been identified as an outer membrane protein (OMP) whose expression is induced upon inorganic phosphate $\left(\mathrm{P}_{\mathrm{i}}\right)$ starvation and requires the binding of $\mathrm{PhoB}$ to the $v c a 1008$ regulatory region (Goulart et al., 2009; von Krüger et al., 2006). A prediction of VCA1008 structure has shown that the protein shares many structural similarities with PhoE of Escherichia coli, a porin also induced under $\mathrm{P}_{\mathrm{i}}$ limitation. Properties such as resistance to denaturation by SDS at lower temperatures and to heat suggest that VCA1008 is a classical trimeric porin, or more precisely the phosphoporin of $V$. cholerae (Goulart et al., 2009). Cells of $V$. cholerae O1 El Tor biotype deficient in VCA1008 expression are less infective in infant mice than those of their parental strain, suggesting an essential role for this protein in the colonization process (Osorio et al., 2004). The observation that vcal008 is expressed during infection in animal models and human volunteers (Lombardo et al., 2007; Osorio et al., 2005) corroborates this idea.

VCA1008 and OmpU are paralogues that share 33\% identity and $62 \%$ similarity, and might play redundant functions in $V$. cholerae cells. Based on these findings we decided to test the hypothesis that the putative phosphoporin VCA1008 could play a role in bile resistance in the pandemic strains N16961SR and O395SR of the El Tor and classical biotypes of of $V$. cholerae O1, respectively.

We demonstrate herein that VCA1008 is required for bile resistance and pathogenicity of N16961SR alone, indicating that the protein is likely to perform distinct functions in classical and El Tor strains of V. cholerae O1. Furthermore, interplay between ToxR- and PhoB/R-dependent regulatory pathways apparently takes place in the El Tor and classical biotypes in a bile salt-dependent and -independent manner, respectively, to control the expression of certain genes and ultimately the physiology and pathogenicity of the bacterium.

\section{METHODS}

Bacterial strains, plasmids and growth conditions. Bacterial strains and plasmids used in this study are listed in Table 1. Strains were routinely grown at $37{ }^{\circ} \mathrm{C}$ in Luria-Bertani broth (LB) or on solid medium [Luria agar (LA), $1.5 \%$ agar in LB]. For growth under defined $\mathrm{P}_{\mathrm{i}}$ concentrations, TG (Tris-glucose with a mix of mineral salts) was

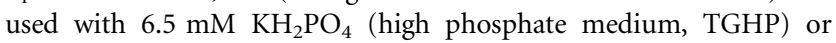

$65 \mu \mathrm{M}$ (low phosphate medium, TGLP; von Krüger et al., 1999). Antibiotics were added to the media at the following concentrations: streptomycin $(\mathrm{Sm}), 100 \mu \mathrm{g} \mathrm{ml}{ }^{-1}$; kanamycin $(\mathrm{Km}), 50 \mu \mathrm{g} \mathrm{m} l^{-1}$; ampicillin (Ap), $100 \mu \mathrm{g} \mathrm{ml}^{-1}$; rifampicin (Rif), $25 \mu \mathrm{g} \mathrm{ml}^{-1}$; chloramphenicol $(\mathrm{Cm}), 20 \mu \mathrm{g} \mathrm{ml}^{-1}$. Because of the high content of $\mathrm{P}_{\mathrm{i}}$ in crude bile, DOC (Sigma) at $0.1-0.2 \%$ was used to mimic bile effects.

Cloning procedures and mutant construction. Genetic manipulations were carried out in E. coli strains $\mathrm{DH} 5 \alpha$ and SY327 $\lambda$ pir. Standard methods were used to prepare plasmids, restriction enzyme digestions and DNA ligations. DNA fragments were purified from agarose gels with a Wizard SV Gel kit (Promega) in accordance with the supplier's recommendations. PCR amplifications were carried out in an automatic thermal cycler (PTC-200, MJ Research) using V. cholerae chromosomal DNA and Taq DNA polymerase (Promega). Amplifications were performed using 35 cycles of denaturation at $94{ }^{\circ} \mathrm{C}$ for $2 \mathrm{~min}$, annealing at $56{ }^{\circ} \mathrm{C}(v c a 1008)$ or $52{ }^{\circ} \mathrm{C}($ tox $R)$ for $1 \mathrm{~min}$, and extension at $72{ }^{\circ} \mathrm{C}$ for $2 \mathrm{~min}$.

For the construction of mutants, fragments containing vca1008 $(1.2 \mathrm{~kb})$ and toxR $(0.9 \mathrm{~kb})$ from $V$. cholerae N16961SR were PCRamplified using the following primers (restriction sites underlined): PhoE1-NcoI (5'-CATAGCCATGGATTCCTCATCACTCACC-3') and PhoERev-SmaI (5'-AAACCCGGGTCGTAACGTAGACCTAG-3'); ToxR-BamHI (5'-CCGGATCCATGTTCGGATTAGGACAC- $\left.3^{\prime}\right)$ and ToxR-HindIII ( $5^{\prime}$-CA $\overline{A G C T T G A C T C A C A C A C C T T G A T G G C-3 ') . ~}$

DNA fragments were cloned into pGEM-T Easy (Promega) to produce pGEM-vca1008 and pGEM-toxR. The plasmids were cut within the vcaloos sequence with EcoRV and within toxR with $\mathrm{NruI}$, where a Km-resistance cassette (1.3 kb HincII fragment of pUC-4K) was inserted to interrupt the target genes.

Plasmids pGEM-vca1008::Km and pGEM-toxR::Km were digested with EcoRI, and the fragments containing the disrupted genes were cloned into the EcoRI site of pGP704. To facilitate mutant selection, the $s a c B$ gene from Bacillus subtilis (1.9 kb EcoRV/BamHI fragment from pJG9) was cloned into both pGP-vca1008::Km and pGPtoxR:: Km, digested with EcoRV and BglII, yielding pGS1 and pGS2, respectively.

Plasmids pGS1 and pGS2 were mobilized into $V$. cholerae $\mathrm{O} 1$ strains

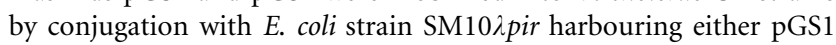
or pGS2. Mutants were obtained by allelic exchange and confirmed by Southern blot analysis, as previously described (von Krüger et al., 1999). Mutants in the vca1008 and toxR genes derived from strain N16961SR were named CG4 and CG5, respectively. The corresponding mutants in O395SR were, respectively, GS1 and GS2 (Table 1).

For complementation analysis, a $1.2 \mathrm{~kb}$ fragment containing the whole vca1008 gene plus an upstream region of $231 \mathrm{bp}$ was PCR-amplified using the primers PhoE1-NcoI (described above) and PhoEStop-SmaI (5'-GCGCCCGGGTTATTAAAAATCGTAACGTAG-3'). The fragment was first cloned into pGEM-T Easy, removed with EcoRI and subcloned into the EcoRI site of pWKS30 to generate pCG15.

To construct a promoter-lac $Z$ fusion we used the promoter-probe vector pIC552. A fragment of $234 \mathrm{bp}$ from the regulatory region upstream of the putative translation start codon of $p h o B$ ( $v c 0719)$ was amplified by PCR using as template chromosomal DNA from $V$. cholerae strain N16961SR and the following primers (endonuclease recognition sites underlined): PhoB-NcoI (5'-CCCCCCATGGTTTAAACCACATTGTTG- $\left.3^{\prime}\right)$ and PhoB-XhoI (5'-TTCTTCTCGAGTGTTTTTCACCAACGCCA- $\left.3^{\prime}\right)$. The fragment was digested with both $\mathrm{NcoI}$ and XhoI and ligated into pIC552 digested with the same restriction endonucleases. It is worth pointing out that in $V$. cholerae strains N16961 and O395, vca1008 and toxR gene sequences plus corresponding regulatory regions are identical. 
Table 1. Bacterial strains and plasmids used in this study

$\mathrm{Ap}^{\mathrm{R}}, \mathrm{Cm}^{\mathrm{R}}, \mathrm{Km}^{\mathrm{R}}, \mathrm{Rif}^{\mathrm{R}}, \mathrm{Sr}^{\mathrm{R}}$ : ampicillin, chloramphenicol, kanamycin, rifampicin and streptomycin resistance, respectively.

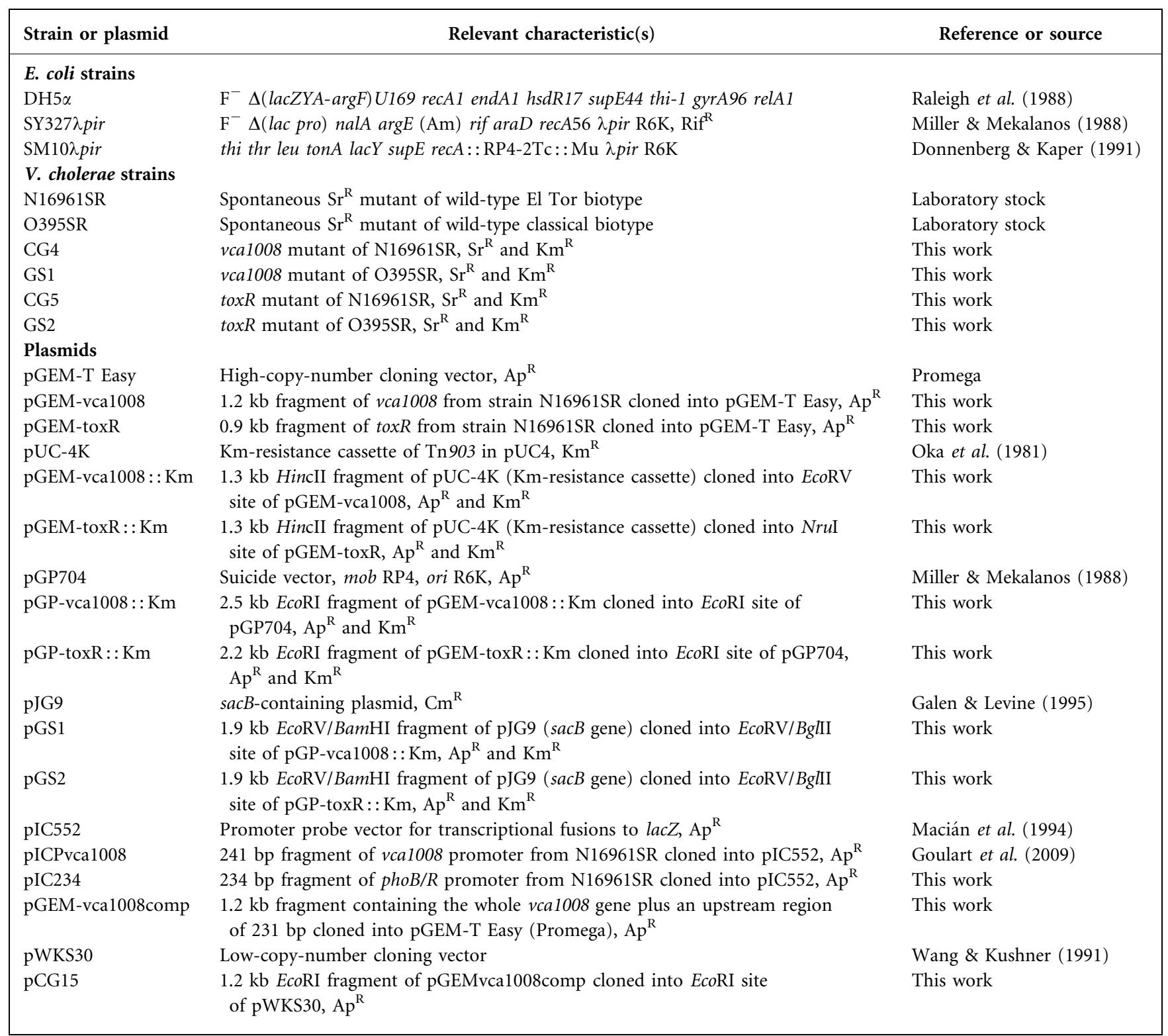

\section{Preparation of outer membrane samples and identification of}

OMPs. OMPs were prepared as previously described (von Krüger et al., 1999). Briefly, cells were disrupted by sonication and cell membranes were recovered after ultracentrifugation $(66000 \mathrm{~g}$ for $40 \mathrm{~min}$ ). The insoluble fraction was first washed with $1 \%$ Sarkosyl and subsequently with $2 \%$ SDS. Insoluble proteins were resuspended in $1 \%$ octyl-polyoxyethylene and analysed by SDS-PAGE. The gels were stained with Coomassie brilliant blue G-250 and proteins were identified by MALDI-TOF analysis, after tryptic digestion, as previously described (von Krüger et al., 2006).

DOC susceptibility analysis. Overnight cultures were diluted $1: 100$ in fresh LB and incubated in a shaking water bath at $37^{\circ} \mathrm{C}$ to early exponential growth phase $\left(\mathrm{OD}_{600} \sim 0.5\right)$. Cells were harvested by centrifugation ( $5000 \mathrm{~g}$ for $5 \mathrm{~min}$ at room temperature), washed, resuspended to the same cell density in TGHP or TGLP, and incubated for $12-14 \mathrm{~h}$ with agitation at $37^{\circ} \mathrm{C}$. Cells were collected by centrifugation, as before, and resuspended in fresh TGHP or TGLP. Viable cell counts (c.f.u.) were determined before (control) and 1, 2 and $5 \mathrm{~min}$ after the addition of $0.2 \%$ DOC to the cultures. The c.f.u. of the control was used as the $100 \%$ value and the survival rates were expressed as percentages of the control. The survival rates of the cells pre-cultivated in TGHP and TGLP were compared using the $t$ test and considered different at $P<0.05$.

Enzymic assays. $\beta$-Galactosidase activities in strains carrying the recombinant plasmids pICPvca1008 and pIC234 were measured as previously described (Goulart et al., 2009). Alkaline phosphatase (PhoA) activity was determined by a variation of the permeabilized whole-cell assay (von Krüger et al., 1999). Activities of both enzymes were expressed in Miller units ( $\mu$ g protein $)^{-1}$, as determined using the Protein Assay Dye Reagent concentrate (Bio-Rad). 
Infant mouse colonization assay. The infant mouse colonization assay was performed as described by Gardel \& Mekalanos (1996). Briefly, V. cholerae cells were grown overnight at $37^{\circ} \mathrm{C}$ in $\mathrm{LB}$ supplemented with the appropriate antibiotics. Infant Swiss albino mice (3-4 days old) were orogastrically inoculated $\left(\sim 10^{6}\right.$ bacteria in $50 \mu \mathrm{l}$ per mouse), separated from their mother and left at $30{ }^{\circ} \mathrm{C}$ for $18 \mathrm{~h}$. A central fraction of the small intestine was removed and homogenized in $2 \mathrm{ml} \mathrm{LB}$. The homogenate was serially diluted and plated onto LA plates containing appropriate antibiotics. For the in vivo competition assay, an equal number of cells was mixed and inoculated (total $\sim 10^{6}$ bacteria in $50 \mu$ per mouse). The total c.f.u. of Km-sensitive (wild-type) and Km-resistant (vca1008 mutant) bacteria was calculated and expressed per gram of tissue. Mean colonization was determined for three replicates with 6-12 animals per experiment.

\section{RESULTS AND DISCUSSION}

\section{Strain-dependent role for vca1008 in growth rate and colonization ability of $V$. cholerae 01}

Disruption of the gene encoding the putative phosphoporin VCA1008 generated mutants that clearly differed from their parental strains. The vca1008 null mutant of N16961SR, strain CG4, presented a reduced growth rate in rich LB medium, but eventually reached a cell density comparable with that of the wild-type strain at the stationary phase (Fig. 1a). Growth deficiency was also observed in TG defined media under both low (TGLP) and high (TGHP) phosphate levels (Fig. 1b, c). These findings correlate well with the reduced intestinal colonization ratio exhibited by the vcalo08 mutant, when tested independently or in the presence of the wild-type strain (Table 2). In contrast to CG4, GS1, the vca1008 null mutant of the classical strain O395SR, grew similarly to the parental strain in either LB or TG medium under high or low levels of $P_{i}$ (Fig. 1). Furthermore, GS1 colonized the infant mouse intestine nearly as efficiently as the wild-type strain when inoculated independently, and out-competed O395SR in mixed-infection assays (Table 2).

These are surprising results, considering that the sequences of genes vca1008 (from the El Tor strain N16961) and VC0395_0230 (from the classical strain O395) and their $250 \mathrm{bp}$ corresponding upstream regions are identical. The distinct phenotypes of vca1008 mutants CG4 and GS1 suggest that this gene might play divergent $\mathrm{P}_{\mathrm{i}}$-independent roles in the physiology and pathogenicity of classical and $\mathrm{El}$ Tor biotypes of $V$. cholerae O1. In addition, they further emphasize the differences between these two strains and suggest that they contrast markedly in the mechanisms whereby they colonize the host intestine.

\section{VCA1008 is required for bile salt resistance in V. cholerae 01 El Tor strain N16961SR}

Earlier studies on $V$. cholerae O1 classical strain O395 have revealed that the ToxR-regulated porin OmpU is critical for bile resistance in vitro (Provenzano et al., 2000; Provenzano \& Klose, 2000). However, this porin is not required for efficient intestinal colonization of animal models by either El Tor or classical strains of $V$. cholerae $\mathrm{O} 1$ (Osorio et al., 2004; Provenzano et al., 2001). The VCA1008 and OmpU genes are paralogues but, in contrast to ompU, vca1008 is essential for intestinal colonization by $V$. cholerae El Tor strains and is expressed in the gastrointestinal tract of animal models and human volunteers (Lombardo et al., 2007; Osorio et al., 2005), where the bacteria encounter bile salts.

These findings lead us to suspect that the putative phosphoporin VCA1008 plays a role in the $V$. cholerae O1 bile resistance mechanism. To investigate this hypothesis, N16961SR and CG4 cells cultivated in both TGLP and
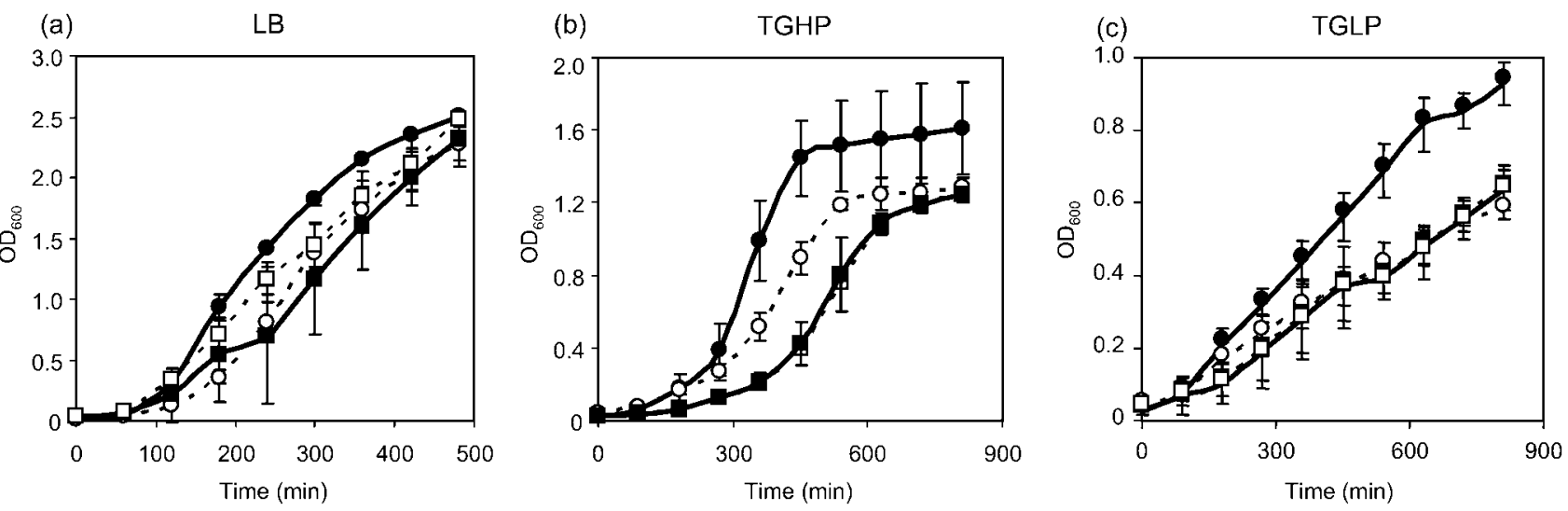

Fig. 1. Growth kinetics of wild-type strains N16961SR and O395SR and their respective isogenic vca1008 mutants CG4 and GS1. V. cholerae cells were grown overnight in LB, inoculated into fresh LB (a), TGHP (b) or TGLP (c) medium and incubated at $37{ }^{\circ} \mathrm{C}$ with aeration for several hours. Growth was monitored by $\mathrm{OD}_{600}$ measurement. Data are representative of three experiments; error bars, SD. Note that time and $\mathrm{OD}_{600}$ scales are different among the three panels. $\bullet, N 16961 S R ; \bigcirc, C G 4$;

口, O395SR; $\square$, GS1. 
Table 2. Colonization efficiency of $V$. cholerae strains

The ratio of wild-type to mutant strains is shown. A ratio greater than 1.0 means that the wild-type colonizes more efficiently than the mutant strain.

\begin{tabular}{|lcc|}
\hline Strains & Independent & Mixed infection \\
\hline N16961SR/CG4 & 5.13 & 17.91 \\
O395SR/GS1 & 1.55 & 0.47 \\
\hline
\end{tabular}

TGHP were subjected to short time exposures (up to $5 \mathrm{~min}$ ) to $0.2 \%$ DOC. Wild-type cells pre-grown in TGLP better tolerated exposure to DOC in the first 2 min than those from TGHP (Fig. 2). The N16961SR vca1008 mutant CG4, on the other hand, presented higher DOC sensitivity than its parental strain, independently of the pre-growth condition (Fig. 2).

Complementation of CG4 with a wild-type vca1008 containing its own regulatory region in plasmid pCG15 restored the ability to survive in the presence of $0.2 \%$ DOC, independently of the $\mathrm{P}_{\mathrm{i}}$ level in the pre-culture medium. Interestingly, CG4/pCG15 cells from TGLP tolerated the bile salt much better in the first 2 min than the wild-type grown under similar conditions. This is probably due to the high copy number of $v c a 1008$ within these cells. However, 5 min later, the CG4/pCG15 DOC survival rate reached that of the parental strain (about $30 \%)$. Taken together, these findings highlight that vca1008 is essential for the survival of N16961SR cells upon exposure to DOC.

To compare the effect of $v c a 1008$ mutation on the bile resistance phenotypes of $V$. cholerae $\mathrm{O} 1$ from distinct biotypes, similar experiments were carried out with cells of the classical strain O395SR and its isogenic vca1008 mutant
GS1. In contrast to N16961SR and CG4 (Fig. 2), O395SR and GS1 cells grown in TGHP were equally sensitive to DOC (Fig. 3). However, a comparison of the survival rates of O395SR and GS1 cells grown in TGLP (Fig. 3) showed that the mutation in $v c a 1008$ affected the bile salt resistance of the classical strain, but not so severely as in the El Tor strain N16961SR (Fig. 2). The higher survival rate of GS1 cells in comparison with CG4 is most likely due to another DOC resistance strategy induced in O395SR cells upon $\mathrm{P}_{\mathrm{i}}$ starvation. Altogether, the above results showed that the vca1008 mutants of the $V$. cholerae $\mathrm{O} 1$ classical and El Tor biotypes differ not only in the ability to colonize the intestinal mucosa of infant mice but also with respect to bile salt resistance and the mechanisms whereby they survive the antimicrobial action of bile salts.

It is well known that outer membrane porins contribute to bile resistance in $V$. cholerae (Provenzano et al., 2001; Provenzano \& Klose, 2000). Hence, to better understand the molecular basis of DOC resistance/sensitivity described above, and to check the involvement of VCA1008 in the process, the porin composition of the wild-type and mutant cells exposed to DOC was analysed. The relatively high DOC resistance of N16961SR cells pre-grown in TGHP could, at least in part, be attributed to OmpU, the major porin in their outer membrane (Fig. 4a). However, OmpU, also the major porin of CG4 cells cultivated in either TGHP or TGLP (Fig. 4a), apparently did not contribute to the cells' resistance to bile, in contrast to earlier results for the classical strain O395 (Provenzano et al., 2000; Provenzano \& Klose, 2000). In fact, as mentioned above, CG4 cells pre-grown in both TGHP and TGLP showed significantly lower bile resistance than the parental strain (Fig. 2). The phosphoporin VCA1008, on the other hand, seems to play an essential role in the bile resistance of the El Tor strain N16961SR (Fig. 2), in perfect

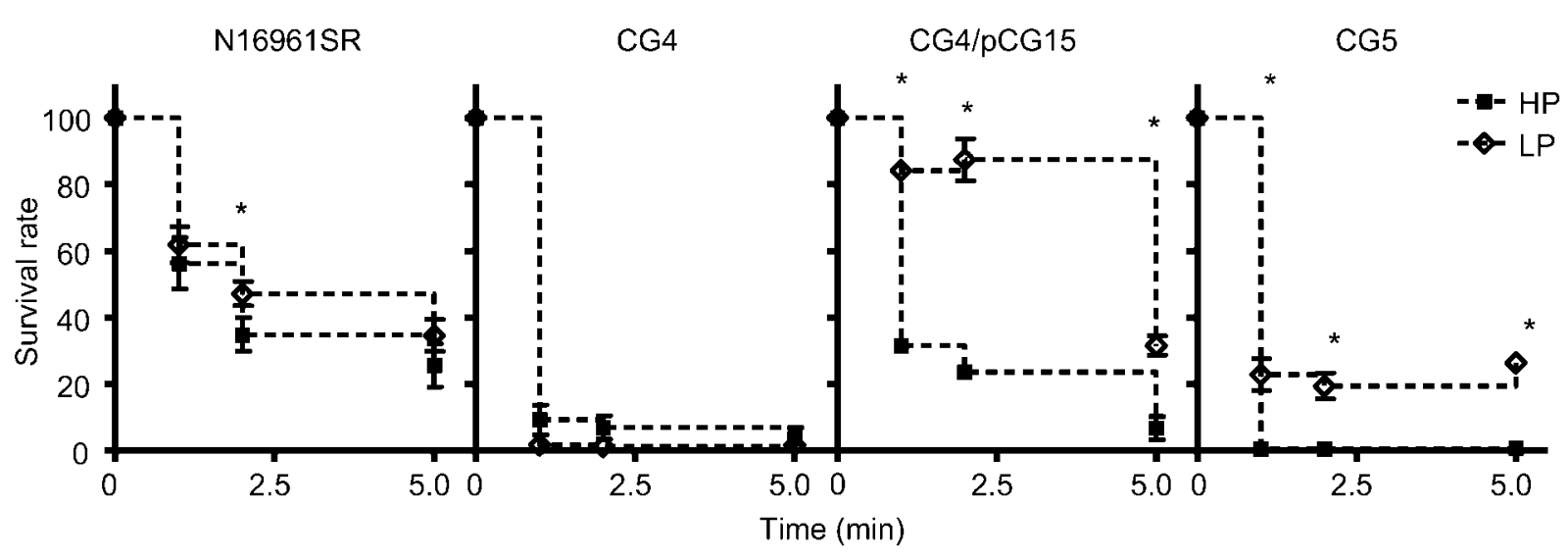

Fig. 2. DOC susceptibility of the wild-type strain N16961SR and vca1008 (CG4) and toxR (CG5) mutants. V. cholerae cells of strains N16961SR, CG4 (vca1008), CG4/pCG15 (complemented in trans with vca1008) and CG5 (toxR) were cultivated in TG medium supplemented with high (HP) or low (LP) $\mathrm{P}_{\mathrm{i}}$ concentrations for $14 \mathrm{~h}$ and then treated with $0.2 \%$ DOC for up to 5 min. Strain CG4/pCG15 presented a wild-type resistance phenotype. Data are representative of three experiments; error bars, SD. Asterisks denote significantly $(P<0.05)$ different survival rates. 


\section{O395SR}

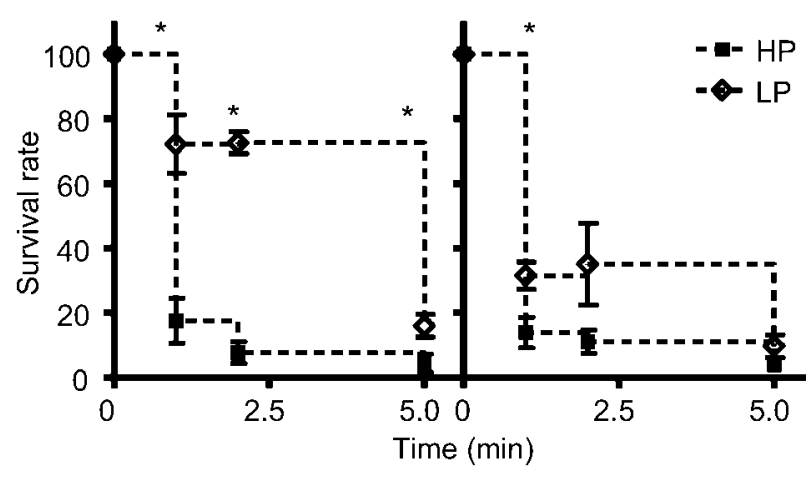

Fig. 3. DOC susceptibility of the wild-type strain O395SR and vca1008 mutant (GS1). O395SR and GS1 cells were cultivated in TG medium with high (HP) or low (LP) $\mathrm{P}_{\mathrm{i}}$ concentrations for $12 \mathrm{~h}$ and then treated with $0.2 \% \mathrm{DOC}$ for up to $5 \mathrm{~min}$. Data are representative of three experiments; error bars, SD. Asterisks denote significantly $(P<0.05)$ different survival rates.

agreement with the reduced intestinal colonization ability of CG4 cells (Table 2).

Strain O395SR and its vca1008 mutant, GS1, grown in TGHP presented OmpT and OmpU as their main porins (Fig. 4b). However, under $P_{i}$ limitation, the parental strain produced VCA1008 and a reduced amount of OmpT, whereas GS1 expressed OmpT as its major porin (Fig. 4b).
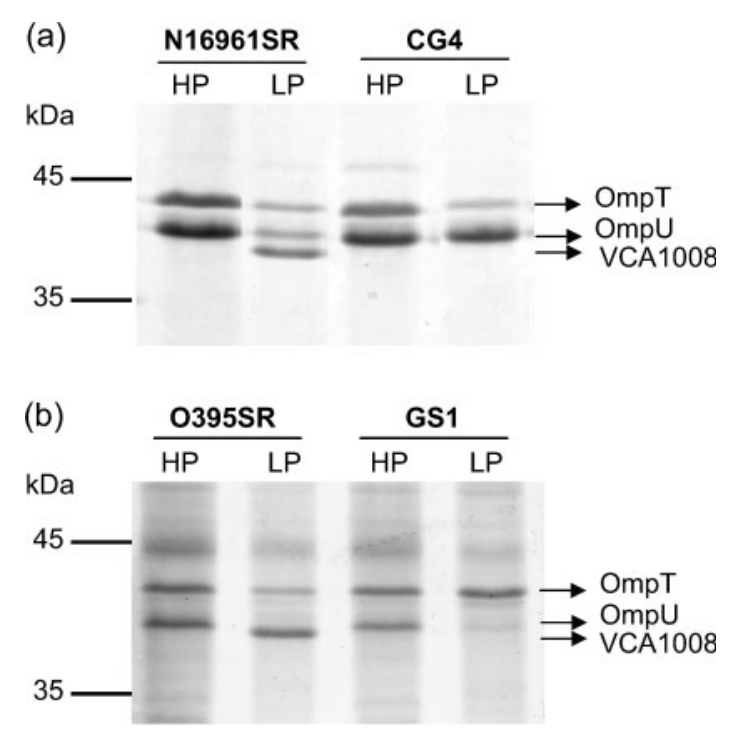

Fig. 4. OMP profiles of El Tor and classical strains and their respective vca1008 mutants grown in TGLP and TGHP. $V$. cholerae El Tor strains N16961SR and CG4 (vca1008) (a), and classical strains O395SR and GS1 (vca1008) (b) were cultivated in TG medium with high (HP) or low (LP) concentrations of $P_{i}$ for $14 \mathrm{~h}$. Their OMP profiles were analysed by $11 \%$ SDS-PAGE and proteins were identified by MS.
Therefore, it is likely that the higher apparent DOC resistance of O395SR cells pre-grown in TGLP in comparison with those from TGHP (Fig. 3) is in part due to the presence of VCA1008 in the outer membrane of the cells.

The fact that cells of both strains, O395SR and GS1, barely expressed $\mathrm{OmpU}$ under $\mathrm{P}_{\mathrm{i}}$-limiting conditions was a great surprise, and might be an indication that $\mathrm{PhoB}$ directly or indirectly represses ompU expression in these strains. Moreover, it is possible that $\mathrm{PhoB}$ activates bile resistance mechanisms in the classical strain other than the modulation of porin expression, which could explain the relatively lower susceptibility to DOC observed in GS1 when compared with the El Tor vca1008 mutant CG4 (Fig. 3).

\section{ToxR-dependent expression of vca1008 and $p h O B / R$ and susceptibility to DOC}

ToxR is a transmembrane transcriptional factor that regulates expression of many known virulence genes of $V$. cholerae $\mathrm{O} 1$ in response to certain environmental cues (reviewed by Childers \& Klose, 2007). It also controls positively and negatively the expression of two major porins, OmpU and OmpT, respectively (Provenzano et al., 2000). However, the mechanisms underlying the ToxR control of outer membrane porin gene expression remain unknown.

Herein, we propose that regulatory pathways controlled by ToxR and PhoB could overlap to modulate the outer membrane porin composition of $V$. cholerae $\mathrm{O} 1$ under $\mathrm{P}_{\mathrm{i}^{-}}$ limiting conditions and, consequently, susceptibility to bile salts. To test this hypothesis, cells of the wild-type N16961SR and CG5, an isogenic toxR mutant, were grown in either TGHP (control condition) or TGLP and their porin composition was analysed. Cells of both strains under $\mathrm{P}_{\mathrm{i}}$ limitation presented VCA1008 as one of their main porins (Figs 4a and 5a), showing that vca1008 expression is apparently not affected by the mutation in toxR under this condition.

The sensitivity to DOC and porin composition of the toxR mutant CG5 were compared with those of N16961SR, as done with CG4. CG5 cells pre-cultivated in TGHP presented OmpT as the major porin (Fig. 5a) and proved to be highly sensitive to DOC (Fig. 2), confirming published results (Provenzano et al., 2000). CG5 cells grown under low $\mathrm{P}_{\mathrm{i}}$ supply, on the other hand, demonstrated DOC resistance comparable with that of N16961SR cells grown in TGHP, after 5 min exposure to the detergent (Fig. 2). In TGLP, the CG5 outer membrane presented VCA1008 and OmpT as major porins (Fig. 5a). As the presence of OmpT does not appear to have a role in DOC resistance, the evidence convincingly suggests that VCA1008 is part of the mechanism of strain CG5 DOC resistance under $\mathrm{P}_{\mathrm{i}}$-limiting conditions.

To further analyse a putative interaction between ToxRand $\mathrm{PhoB} / \mathrm{R}$-dependent regulatory pathways, the activity of the $v c a 1008$ promoter was evaluated in the toxR mutant CG5 and in the wild-type N16961SR cells under high and 
(a)

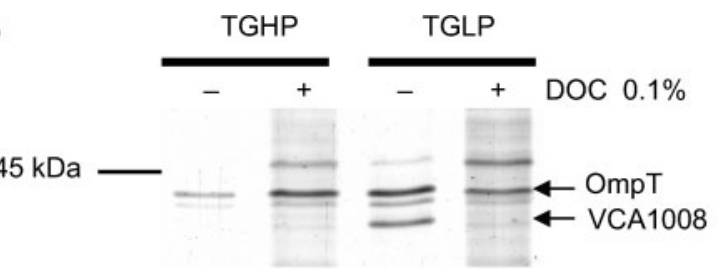

(b)

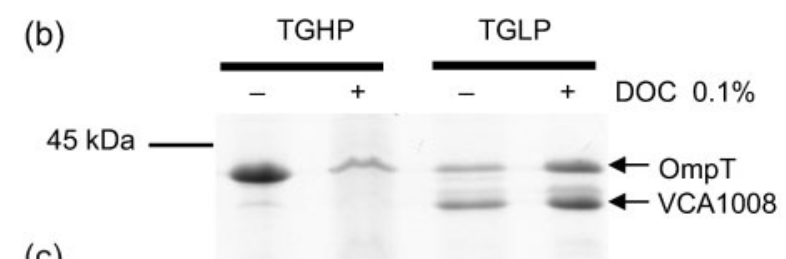

(c)

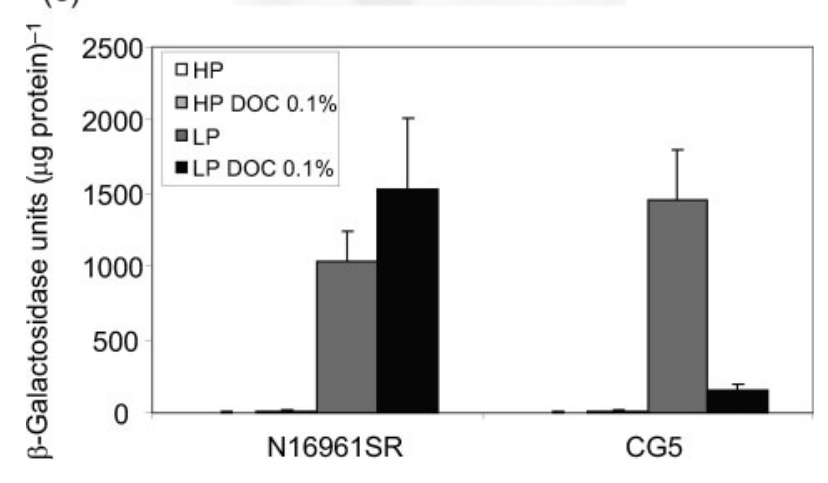

Fig. 5. Effect of DOC on OMP profiles of toxR mutants of El Tor and classical strains and on the activity of the vca1008 promoter in $\mathrm{N} 16961 \mathrm{SR}$ and in its toxR mutant CG5. Cells were grown for $14 \mathrm{~h}$ in TG medium with high (HP) or low (LP) levels of $P_{i}$ in the presence or absence of $0.1 \%$ DOC. OMP profiles were analysed by $11 \%$ SDS-PAGE and proteins were identified by MS: OMPenriched samples of the El Tor toxR mutant CG5 (a) and classical toxR mutant GS2 (b). (c) $\beta$-Galactosidase activity measured in wild-type N16961SR and toxR mutant CG5 strains transformed with plCPvca1008 (containing the vca1008 promoter fused to lacZ) grown as described above.

low levels of $\mathrm{P}_{\mathrm{i}}$, in the absence and presence of $0.1 \%$ DOC. The detergent was added to the media because it is well known that the ToxR transcriptional activity in both biotypes of $V$. cholerae O1, El Tor and classical, is modulated by bile salts (Provenzano et al., 2000). Moreover, we hypothesized that some of these in vitro conditions mimic aspects of the intestinal environment. These are the presence of bile salts, $\mathrm{pH}$ above $7.0,37^{\circ} \mathrm{C}$ and $\mathrm{P}_{\mathrm{i}}$ limitation, since it has been observed that some $V$. cholerae $\mathrm{PhoB} / \mathrm{R}$-regulated genes are induced in vivo (Merrell et al., 2002; Osorio et al., 2004, 2005).

The putative regulatory region of $v c a 1008$ was fused to $l a c Z$ in a low copy plasmid (pICPvca1008) and its promoter activity was evaluated in N16961SR and CG5 cells grown as described above. Virtually no $\beta$-galactosidase activity was detected in the cells of either strain transformed with pICPvca1008 and grown in TGHP in either the absence or presence of DOC (Fig. 5c). Similar results were obtained with cells harbouring the promoterless pIC552 (control; data not shown).

In contrast, a high level of $\beta$-galactosidase activity was observed in N16961SR/pICPvca1008 cells grown in TGLP, and even higher lac $Z$ expression (1.5-fold the expression in TGLP) was detected in cells cultivated in TGLP/DOC (Fig. 5c). This result indicates that DOC increased the transcription rate from the vcal008 promoter in the wild-type cells under $\mathrm{P}_{\mathrm{i}}$ limitation. However, the vca1008 promoter activity was 1.4-fold higher in CG5 than in N16961SR in TGLP, suggesting a negative control of vca1008 expression by ToxR, under low $\mathrm{P}_{\mathrm{i}}$ supply (Fig. 5c). Taken together, these findings show that under $\mathrm{P}_{\mathrm{i}}$-limiting conditions, ToxR apparently reduces the expression of the porin gene $v c a 1008$, but in TGLP in the presence of DOC its expression is enhanced.

In CG5/pICPvca1008 cells grown in TGLP, $\beta$-galactosidase activity was high and comparable with that in N16961SR cultured in TGLP/DOC (Fig. 5c). Surprisingly, however, the activity of $\beta$-galactosidase in cells of CG5/pICPvca1008 cultivated in TGLP/DOC was 10 -fold lower than in the same cells grown in TGLP and also in N16961SR/ pICPvca1008 cultivated in TGLP/DOC (Fig. 5c). This result confirms the above observation that ToxR/DOC indeed has a positive effect on the expression of vca1008 in N16961SR.

Curiously, the bile salt seems to inhibit the activation of the vca1008 promoter by $\mathrm{PhoB}$, otherwise its activity level in CG5 in TGLP/DOC would have been similar to that observed in the cells grown in TGLP alone (Fig. 5c). Accordingly, the low activation of the $v c a 1008$ promoter in CG5/pICPvca1008 in TGLP/DOC (Fig. 5c) correlates well with the negligible amount/absence of VCA1008 in the outer membrane of these cells (Fig. 5a).

Altogether, these results suggest that in N16961SR under $\mathrm{P}_{\mathrm{i}}$-limiting conditions, vca1008 expression is under the control of the transcription factors PhoB and ToxR, whose activities towards the gene promoter are modulated by the bile salt. Under $\mathrm{P}_{\mathrm{i}}$ limitation (TGLP), vcalo08 promoter activation by PhoB seems to overcome the negative effect of ToxR on the promoter (Fig. 5c) and a high amount of VCA1008 is produced (Fig. 4). However, when DOC is added to the medium (TGLP/DOC), the PhoB-mediated activation of $v c a 1008$ expression is negatively affected, although vca1008 expression via ToxR is enhanced. How PhoB and ToxR/bile salt interact to control vca1008 expression is not known. Nevertheless, it is apparent that $\mathrm{PhoB}$ is required in vitro for the ToxR/DOC-mediated mechanism of regulation of $v c a 1008$ expression, since it was only observed under $\mathrm{P}_{\mathrm{i}}$-limiting conditions.

VCA1008 expression was also evaluated in the toxR mutant GS2, derived from the classical strain O395SR (Fig. 5b). Contrary to the results observed for the El Tor strain CG5, disruption in the toxR gene did not affect VCA1008 
biosynthesis in GS2 cells under $\mathrm{P}_{\mathrm{i}}$ limitation in the presence of DOC. These findings clearly demonstrated that regulation of vca1008 expression is different in classical and $\mathrm{El}$ Tor strains of $V$. cholerae $\mathrm{O} 1$.

To investigate whether the increased expression of $v c a 1008$ in the presence of bile salt is correlated with alterations in the expression of other Pho regulon genes, the activity of the promoter of the $p h o B / R$ operon (fused to lacZ in the low copy plasmid pIC234) was determined in N16961SR and CG5 cells grown in TGLP and TGLP/DOC (Fig. 6).

Similarly to the results observed for the $v c a 1008$ promoter (Fig. 5c), the activity of the $p h o B / R$ promoter was 1.4 -fold higher in CG5 than in N16961SR in TGLP, suggesting a negative control of $p h o B / R$ expression by ToxR, under low $P_{\mathrm{i}}$ supply (Fig. 6a). Moreover, the activity of the $p h o B / R$ promoter in each strain was reduced in TGLP/DOC in comparison with TGLP (Fig. 6a), indicating that the expression of the $p h o B / R$ operon is negatively affected by the bile salt under $\mathrm{P}_{\mathrm{i}}$ limitation. Interestingly, the effect of

(a)

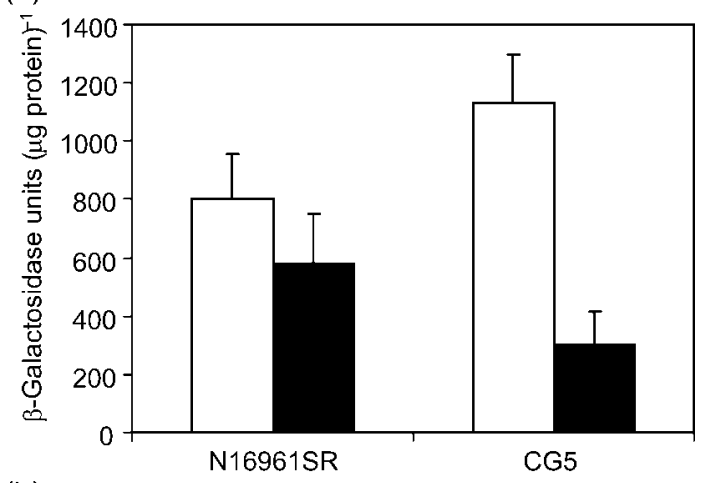

(b)

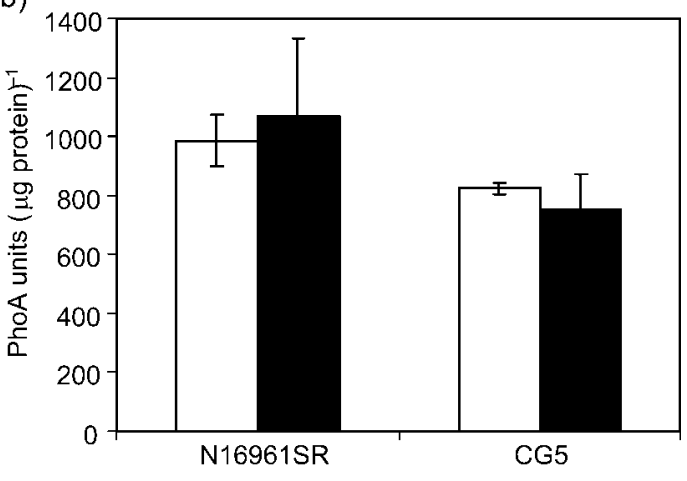

Fig. 6. Effect of DOC on the expression of the Pho regulon (PhoA) and on the activity of the phoB/R promoter in N16961SR and its toxR mutant CG5 grown in low- $\mathrm{P}_{\mathrm{i}}$ medium. Cells were grown for $14 \mathrm{~h}$ in TGLP in the absence (white bars) or presence of $0.1 \%$ DOC (black bars). (a) $\beta$-Galactosidase activities were measured in N16961SR and toxR mutant CG5 transformed with pIC234 (containing the $p h o B / R$ promoter fused to lacZ). (b) PhoA activities were measured in the same cells. Data are representative of three independent experiments; error bars, SD.
DOC on $p h o B / R$ promoter activity was greater in the tox $R$ mutant than in the wild-type, leading us to believe that ToxR/DOC acts synergistically to upregulate the expression of $p h o B / R$, as observed for $v c a 1008$ (Fig. 5c). Taken together, these findings prompted us to suggest that in TGLP, the expression of $p h o B / R$ in N16961SR cells is controlled positively by PhoB and negatively by a ToxRdependent pathway. DOC somehow changes the balance between these regulatory systems and ultimately their activity towards the $p h o B / R$ promoter, with a consequent alteration in the operon expression in N16961SR under $\mathrm{P}_{\mathrm{i}}{ }^{-}$ limited conditions.

In this scenario, lower levels of $\mathrm{PhoB}$ might be expected in N16961SR cells cultivated in TGLP/DOC than in those cultivated in TGLP. Hence, the increased activation of the vca1008 promoter in N16961SR cells cultured in TGLP/ DOC (Fig. 5c), in a background of reduced $p h o B / R$ expression, might indeed be due to the positive effect of ToxR/DOC, also observed with respect to the $p h o B / R$ promoter, but at a lower level (Fig. 6a).

PhoA, a protein with alkaline phosphatase activity, is also induced in V. cholerae cells under $\mathrm{P}_{\mathrm{i}}$ limitation (von Krüger et al., 1999) and is considered a natural reporter of the Pho regulon. Thus, to evaluate expression of the regulon in N16961SR and CG5, PhoA activity was measured in the cells grown under $\mathrm{P}_{\mathrm{i}}$ limitation, in either the presence or absence of DOC. PhoA activity was detected in both N16961SR and CG5 cells, and did not change by addition of DOC to the culture medium. However, in the toxR mutant cells, PhoA activity was slightly lower (about $20 \%$ ) than in N16961SR cells (Fig. 6b). Putting our results together, we conclude that another regulatory molecule/ system besides $\mathrm{PhoB}$ is involved in the regulation of $v c a 1008$ and phoB/R expression in $V$. cholerae O1 strain N16961SR, and that ToxR appears to be the most suitable candidate. Further studies are under way to determine whether ToxR interacts directly with the regulatory regions of $v c a 1008$ and $p h o B / R$, or whether some other indirect mechanism is involved.

\section{Concluding remarks}

This study revealed many surprising findings on the gene regulation, physiology and pathogenicity of the pandemic strains of V. cholerae O1, N16961SR and O395SR, of the El Tor and classical biotypes, respectively. As far as we know this is the first report of an interplay between ToxR and PhoB regulatory systems to control porin gene expression, of the influence of DOC on the expression levels of PhoBand ToxR-regulated genes, and of the requirement for the putative phosphoporin VCA1008 for bile resistance in $V$. cholerae.

In the El Tor strain N16961SR, ToxR and PhoB regulatory systems seem to control in a bile salt-dependent manner the expression of vca1008, whose product is absolutely required for the bile resistance and pathogenicity of the 
bacterium. The same was not observed with strain O395SR, indicating that the classical and El Tor strains differ markedly with respect to the strategies that they use to deal with the toxic effects of bile in vitro and, most likely, in vivo as well, to cause cholera. The classical and El Tor strains also seem to differ in the regulation of OmpU expression under $\mathrm{P}_{\mathrm{i}}$ limitation. Under such conditions the cells of classical strains present high levels of VCA1008 and reduced amounts of OmpU. The molecular mechanisms behind ompU gene downregulation are not known, but they might involve a synergy between ToxR- and PhoBdependent regulatory pathways. As far as is known, neither OmpU nor VCA1008 is essential to the pathogenicity of classical strains of $V$. cholerae O1. Therefore, a role for this particular bile salt-independent ToxR-PhoB system interaction in $V$. cholerae O1 O395SR in vivo is not clear. However, it is possible that in the natural habitat, where the $\mathrm{P}_{\mathrm{i}}$ supply is limited, members of the ToxR and PhoB regulons team up to upregulate $v c a 1008$ and downregulate ompU, as a strategy to increase the $\mathrm{P}_{\mathrm{i}}$-uptake rate of $V$. cholerae $\mathrm{O} 1$ cells.

The data presented herein also permit us to speculate that upon infection of the intestinal tract by N16961SR, bile salts somehow modulate the transcription rate of the phoB/ $R$ operon and the transcriptional activity of the regulators PhoB and ToxR to allow the appropriate expression of genes required for bile resistance and virulence. The molecular mechanisms by which bile salts control ToxRand PhoB-regulated genes are not clear, and how the low $\mathrm{P}_{\mathrm{i}}$-induced porin protects $V$. cholerae $\mathrm{O} 1$ cells from the cytotoxic effects of bile remains to be found. Therefore, further work will be needed to clarify these intriguing and exciting issues, which could greatly contribute to our understanding of the regulatory mechanisms involved in the gut $-V$. cholerae interaction.

\section{ACKNOWLEDGEMENTS}

We are grateful to Mr Celso Pereira, Mr Eduardo Camacho and Mrs Lílian A. Sá for technical support, and to the Proteomics Network of Rio de Janeiro for the use of MALDI-TOF. We thank Mrs J. Farache for pIC234, and Fundação Carlos Chagas Filho de Amparo à Pesquisa do Estado do Rio de Janeiro (FAPERJ), Conselho Nacional de Desenvolvimento Científico e Tecnológico $(\mathrm{CNPq})$ and Coordenação de Aperfeiçoamento de Pessoal de Nível Superior (CAPES) for financial support.

\section{REFERENCES}

Achouak, W., Heulin, T. \& Pages, J. M. (2001). Multiple facets of bacterial porins. FEMS Microbiol Lett 199, 1-7.

Begley, M., Gahan, C. G. M. \& Hill, C. (2005). The interaction between bacteria and bile. FEMS Microbiol Rev 29, 625-651.

Bernstein, C., Bernstein, H., Payne, C. M., Beard, S. E. \& Schneider, J. (1999). Bile salt activation of stress response promoters in Escherichia coli. Curr Microbiol 39, 68-72.

Bina, J. E. \& Mekalanos, J. J. (2001). Vibrio cholerae tolC is required for bile resistance and colonization. Infect Immun 69, 4681-4685.
Chatterjee, A., Chaudhuri, S., Saha, G., Gupta, S. \& Chowdhury, R. (2004). Effect of bile on the cell surface permeability barrier and efflux system of Vibrio cholerae. J Bacteriol 186, 6809-6814.

Childers, B. M. \& Klose, K. E. (2007). Regulation of virulence in Vibrio cholerae: the ToxR regulon. Future Microbiol 2, 335-344.

Chowdhury, R., Sahu, G. K. \& Das, J. (1996). Stress response in pathogenic bacteria. J Biosci 21, 149-160.

Donnenberg, M. S. \& Kaper, J. B. (1991). Construction of an eae deletion mutant of enteropathogenic Escherichia coli by using a positive-selection suicide vector. Infect Immun 59, 4310-4317.

Duret, G. \& Delcour, A. H. (2006). Deoxycholic acid blocks Vibrio cholerae OmpT but not OmpU porin. J Biol Chem 281, 19899-19905.

Galen, J. E. \& Levine, M. M. (1995). Improved suicide vector for chromosomal mutagenesis in Salmonella typhi. In Abstracts of the 95th General Meeting of the American Society for Microbiology, p. 158. Washington, DC: American Society for Microbiology.

Gänzle, M. G., Hertel, C., van der Vossen, J. M. B. M. \& Hammes, W. P. (1999). Effect of bacteriocin-producing lactobacilli on the survival of Escherichia coli and Listeria in a dynamic model of the stomach and the small intestine. Int J Food Microbiol 48, 21-35.

Gardel, C. L. \& Mekalanos, J. J. (1996). Alterations in Vibrio cholerae motility phenotypes correlate with changes in virulence factor expression. Infect Immun 64, 2246-2255.

Goulart, C. L., Lery, L. M. S., Diniz, M. M. P., Vianez, J. L., NevesFerreira, A. G. C., Perales, J., Bisch, P. M. \& von Krüger, W. M. A. (2009). Molecular analysis of VCA1008: a putative phosphoporin of Vibrio cholerae. FEMS Microbiol Lett 298, 241-248.

Gunn, J. S. (2000). Mechanisms of bacterial resistance and response to bile. Microbes Infect 2, 907-913.

Gupta, S. \& Chowdhury, R. (1997). Bile affects production of virulence factors and motility of Vibrio cholerae. Infect Immun 65, 1131-1134.

Krukonis, E. S. \& Dirita, V. J. (2003). From motility to virulence: sensing and responding to environmental signals in Vibrio cholerae. Curr Opin Microbiol 6, 186-190.

Leverrier, P., Dimova, D., Pichereau, V., Auffray, Y., Boyaval, P. \& Jan, G. L. (2003). Susceptibility and adaptive response to bile salts in Propionibacterium freudenreichii: physiological and proteomic analysis. Appl Environ Microbiol 69, 3809-3818.

Lombardo, M. J., Michalski, J., Martinez-Wilson, H., Morin, C., Hilton, T., Osorio, C. G., Nataro, J. P., Tacket, C. O., Camilli, A. \& other authors (2007). An in vivo expression technology screen for Vibrio cholerae genes expressed in human volunteers. Proc Natl Acad Sci U S A 104, 18229-18234.

Macián, F., Perezroger, I. \& Armengod, M. E. (1994). An improved vector system for constructing transcriptional lacZ fusions - analysis of regulation of the $d n a A, d n a N, r e c F$ and $g y r B$ genes of Escherichia coli. Gene 145, 17-24.

Merrell, D. S., Hava, D. L. \& Camilli, A. (2002). Identification of novel factors involved in colonization and acid tolerance of Vibrio cholerae. Mol Microbiol 43, 1471-1491.

Miller, V. L. \& Mekalanos, J. J. (1988). A novel suicide vector and its use in construction of insertion mutations - osmoregulation of outer membrane proteins and virulence determinants in Vibrio cholerae requires toxR. J Bacteriol 170, 2575-2583.

Oka, A., Sugisaki, H. \& Takanami, M. (1981). Nucleotide sequence of the kanamycin resistance transposon Tn903. J Mol Biol 147, 217-226.

Osorio, C. G., Martinez-Wilson, H. \& Camilli, A. (2004). The ompU paralogue vcalo08 is required for virulence of Vibrio cholerae. J Bacteriol 186, 5167-5171. 
Osorio, C. G., Crawford, J. A., Michalski, J., Martinez-Wilson, H., Kaper, J. B. \& Camilli, A. (2005). Second-generation recombinationbased in vivo expression technology for large-scale screening for Vibrio cholerae genes induced during infection of the mouse small intestine. Infect Immun 73, 972-980.

Pace, J. L., Chai, T. J., Rossi, H. A. \& Jiang, X. P. (1997). Effect of bile on Vibrio parahaemolyticus. Appl Environ Microbiol 63, 2372-2377.

Plésiat, P. \& Nikaido, H. (1992). Outer membranes of Gram-negative bacteria are permeable to steroid probes. Mol Microbiol 6, 1323-1333.

Poole, K. (2002). Outer membranes and efflux: the path to multidrug resistance in Gram-negative bacteria. Curr Pharm Biotechnol 3, 77-98.

Provenzano, D. \& Klose, K. E. (2000). Altered expression of the ToxR-regulated porins OmpU and OmpT diminishes Vibrio cholerae bile resistance, virulence factor expression, and intestinal colonization. Proc Natl Acad Sci U S A 97, 10220-10224.

Provenzano, D., Schuhmacher, D. A., Barker, J. L. \& Klose, K. E. (2000). The virulence regulatory protein ToxR mediates enhanced bile resistance in Vibrio cholerae and other pathogenic Vibrio species. Infect Immun 68, 1491-1497.

Provenzano, D., Lauriano, C. M. \& Klose, K. E. (2001). Characterization of the role of the ToxR-modulated outer membrane porins OmpU and OmpT in Vibrio cholerae virulence. J Bacteriol 183, 3652-3662.

Raleigh, E. A., Murray, N. E., Revel, H., Blumenthal, R. M., Westaway, D., Reith, A. D., Rigby, P. W. J., Elhai, J. \& Hanahan, D. (1988). $m c r A$ and $m c r B$ restriction phenotypes of some Escherichia coli strains and implications for gene cloning. Nucleic Acids Res 16, 1563-1575.

Reidl, J. \& Klose, K. E. (2002). Vibrio cholerae and cholera: out of the water and into the host. FEMS Microbiol Rev 26, 125-139.

Savijoki, K., Suokko, A., Palva, A., Valmu, L., Kalkkinen, N. \& Varmanen, P. (2005). Effect of heat-shock and bile salts on protein synthesis of Bifidobacterium longum revealed by [S-35]methionine labelling and two-dimensional gel electrophoresis. FEMS Microbiol Lett 248, 207-215.

Simonet, V. C., Basle, A., Klose, K. E. \& Delcour, A. H. (2003). The Vibrio cholerae porins $\mathrm{OmpU}$ and OmpT have distinct channel properties. J Biol Chem 278, 17539-17545.

Snyder, D. S. \& McIntosh, T. J. (2000). The lipopolysaccharide barrier: correlation of antibiotic susceptibility with antibiotic permeability and fluorescent probe binding kinetics. Biochemistry 39, 11777-11787.

Thanassi, D. G., Cheng, L. W. \& Nikaido, H. (1997). Active efflux of bile salts by Escherichia coli. J Bacteriol 179, 2512-2518.

van Velkinburgh, J. C. \& Gunn, J. S. (1999). PhoP-PhoQ-regulated loci are required for enhanced bile resistance in Salmonella spp. Infect Immun 67, 1614-1622.

von Krüger, W. M. A., Humphreys, S. \& Ketley, J. M. (1999). A role for the PhoBR regulatory system homologue in the Vibrio cholerae phosphate limitation response and intestinal colonization. Microbiology 145, 24632475.

von Krüger, W. M. A., Lery, L. M. S., Soares, M. R., Neves-Manta, F. S., Silva, C. M. B. E., Neves-Ferreira, A. G. D., Perales, J. \& Bisch, P. M. (2006). The phosphate-starvation response in Vibrio cholerae $\mathrm{O} 1$ and $p h o B$ mutant under proteomic analysis: disclosing functions involved in adaptation, survival and virulence. Proteomics 6, 14951511.

Wai, S. N., Mizunoe, Y. \& Yoshida, S. (1999). How Vibrio cholerae survive during starvation. FEMS Microbiol Lett 180, 123-131.

Wang, R. F. \& Kushner, S. R. (1991). Construction of versatile lowcopy-number vectors for cloning, sequencing and gene expression in Escherichia coli. Gene 100, 195-199.

Edited by: D. M. Gordon 